\title{
Estimated Performance of Nanoscopium at an Upgraded Synchrotron Soleil.
}

\author{
A. Somogyi $^{1 *}$, G. Baranton ${ }^{1}$, K. Medjoubi ${ }^{1}$ \\ 1. Nanoscopium, Sychrotron Soleil, St Aubin and France. \\ * Corresponding author, somogyi@synchrotron-soleil.fr.
}

At the dawn of third generation synchrotron source upgrades the question of analytical performance of existing scanning X-ray nanoprobes at an upgraded source is of significant importance; what is the cost/performance/time-line figure-of-merit that they fully profit from the increased source brilliance? Synchrotron Soleil (France) considers the source upgrade to achieve near-diffraction limited performance up to the hard X-ray range. The present Soleil source is partially coherent; the vertical and horizontal beam properties are very different, the horizontal source size and divergence is much larger than the diffraction limit. The Nanoscopium hard X-ray nanoprobe beamline is dedicated to hierarchical length-scale fast scanning multi-technique imaging [1]. It has been designed for performing flux-versusresolution trade-off by selecting one or multiple coherence modes by its secondary source (SS) aperture. This also permits to filter out beamline-optics-caused instabilities. By combining both diffractive and reflective optics, both "flux- and coherence-hungry" applications could be implemented successfully in the 5-20 keV energy range. The spatial resolution [30 nm, $300 \mathrm{~nm}$ ] and the scanned field-of-view can be easily adapted to the needs of the analytical techniques and scientific applications. The beamline offers simultaneous information on sample morphology by exploiting scanning X-ray microscopy techniques, coherent imaging and recently fast full field tomography [K. Medjoubi et al, this conference].

A crucial requirement of a multi-technique nano-probe with spectro-microscopy capabilities is the easy and fast adaptation of the energy without beamline realignment. This is facilitated by using achromatic nano-focusing optic. Such optic, a total-reflection Kirkpatrick-Baez (KB) mirror was chosen for an endstation of the beamline, which is situated at $70 \mathrm{~m}$ from the SS. KB mirrors have minimal chromatic aberration and total reflection KB's can accept and focus the full bandpass of the insertion device with high reflectivity. However, their numerical aperture (NA) is limited by the incidence angle and the mirror length (Fig. 1). A large-NA, bulky KB mirror necessitates a relatively large, $\geq 100 \mathrm{~mm}$ working distance between the second mirror and the sample. Nanoscopium opted for $\sim 100 \mathrm{~mm}$ long Rh-coated mirrors with $3 \mathrm{mrad}$ total reflection angle. The $130 \mathrm{~mm}$ working distance is an advantage for introducing different sample environments for in-situ capabilities. Diffraction-limited focusing can be reached if both the beam size and the energy dependent transverse coherence length at the KB aperture are larger than its effective aperture [2]. This can be easily achieved in the 5-20 keV energy range by tuning the high precision SS in the $\geq 5 \mu \mathrm{m}$ range. The high optical quality KB (JTEC) reaches diffraction limited focusing corresponding to its design parameters ( 60 $\mathrm{nm} \mathrm{FWHM}$ at $15 \mathrm{keV})$ [1]. The high position and angular stability of the focused nano-beam is crucial in the $1 \mathrm{~Hz}-1 \mathrm{kHz}$ frequency range, corresponding to usual sampling frequencies of multi-length-scale imaging. The SS permits to filter out the positional and angular instabilities of the X-ray beam. The typical mechanical instabilities of beamline optics is in the $<50 \mathrm{~Hz}$ range. The noise power spectrum of the position of the transmitted beam measured with a XPAD pixel-detector at $2.5 \mathrm{~m}$ behind the $\mathrm{KB}$ mirror is shown in Figure 2. It illustrates the spatial filtering effect of the SS. At $10 \mathrm{keV}$ the vibration peaks caused by the pre-focusing optic between 25.3 and $38.5 \mathrm{~Hz}$ 's are measured at $30 \mu \mathrm{m} \mathrm{SS}$ opening (red dotted curve) but they disappear at $<10 \mu \mathrm{m}$ SS (blue curve), which corresponds to the coherent illumination of the KB. Thus, importantly, the SS significantly reduces beam vibration induced by the pre-focusing beamline optics of Nanoscopium. 


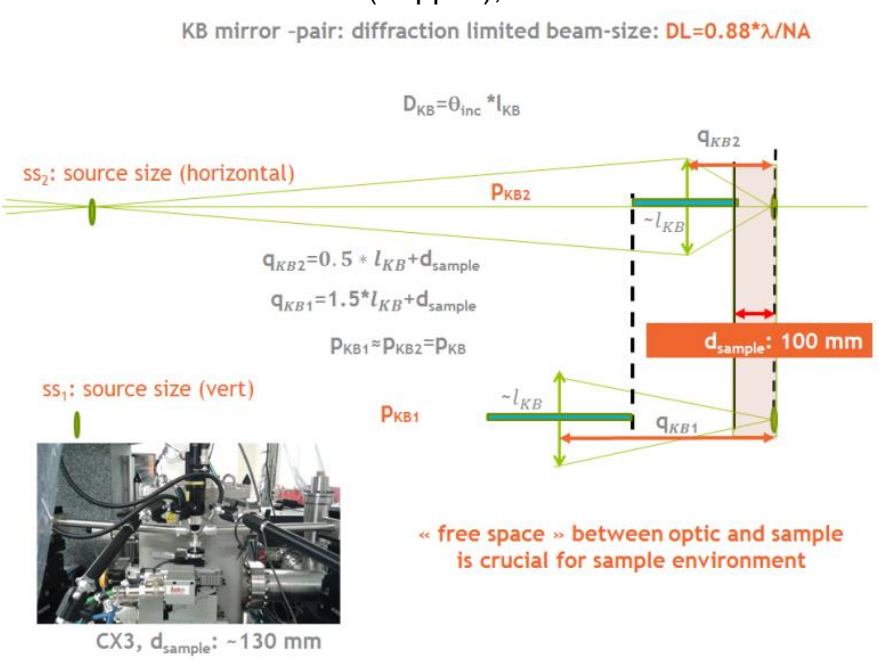

Figure. 1. Scheme of KB-based nanofocusing. Inset: KB-based end-station of Nanoscopium

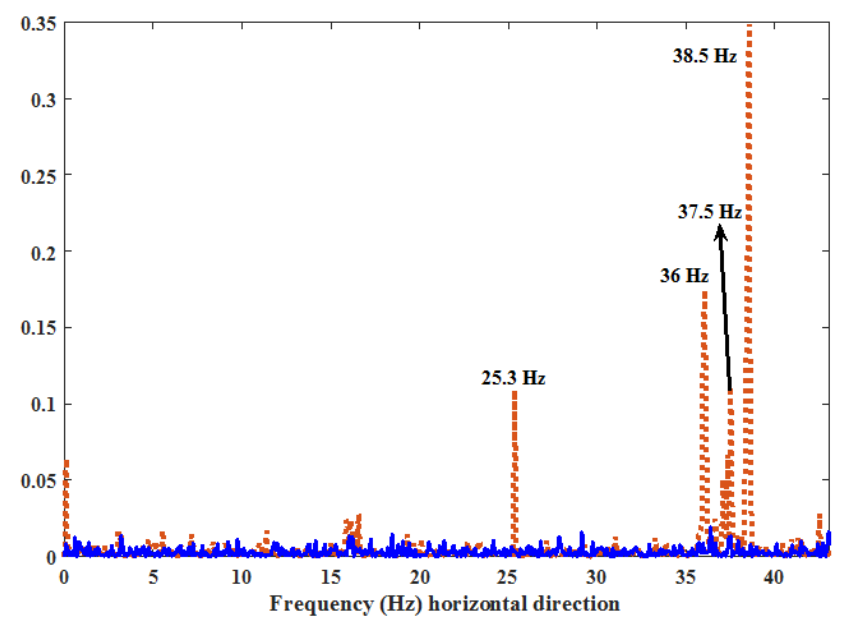

Figure. 2. Noise power spectrum of the beam position after the $\mathrm{KB}$ at $10 \mathrm{keV}$. Red dotted curve: $\mathrm{SS}=30 \mu \mathrm{m}$, blue curve: $\mathrm{SS}=10 \mu \mathrm{m}$.

In general, the beamline design of a KB-based nanoprobe at a diffraction limited storage ring (DLSR) will be governed by the smallest achievable diffraction limited beam-size squeezing the full achievable coherent flux [3,4]. Clearly, the diffraction-limited focusing is very inefficient at the present Soleil Source; due to the low horizontal brilliance only a small, $<1 \%$ fraction of the horizontal X-ray beam can be transferred into the diffraction limited focus. The performance of the Nanoscopium beamline at the upgraded Soleil source can be estimated by considering the improved source performance. A future lowemittance Soleil source will produce at least a fiftyfold increase in the horizontal flux density at the secondary source of Nanoscopium, which will be directly transferred into the flux density of the nanobeam. For diffraction limited focusing, both the beam size $\Sigma_{\mathrm{h} / \mathrm{v}}$ and lateral coherence length $1_{\text {coh,h/v }}$ must be larger at the KB position than the effective $\mathrm{KB}$ aperture $\mathrm{D}_{\mathrm{KB}}$. The $\mathrm{SS}$ of the beamline can be readily tuned to fulfil both of these criteria with $2 * 1_{\mathrm{coh}, \mathrm{h} / \mathrm{v}}>\mathrm{D}_{\mathrm{KB}}$ when the full coherent beam fraction can be focused to the diffraction limit. The $\mathrm{p}_{\mathrm{KB}} \sim 70 \mathrm{~m}$ SS-KB distance assures that the $\mathrm{SS}^{*} \mathrm{q}_{\mathrm{KB}} / \mathrm{p}_{\mathrm{KB}}$ demagnification does not give significant contribution to the diffraction limited beam-size in case of coherent illumination of the KB. Moreover, with the recent optical beamline design and distances, 30 $\mathrm{nm}$ diffraction limited focus could be reached with an updated $\mathrm{KB}$ at $20 \mathrm{keV}$ while keeping $\sim 100 \mathrm{~mm}$ working distance.

As a conclusion, Nanoscopium is well adapted to a future Soleil source upgrade without major beamline modification. It will readily and directly profit from the improved higher source brilliance providing 30$300 \mathrm{~nm}$ sized beams with > hundredfold flux increase within a very short time-frame after a future Soleil source upgrade. Its two-stage optical design scheme relaxes the X-ray source stability requirements and is optimal to match the experimental requirements at such a multi-technique hierarchical length-scale imaging beamline without compromising easy beamline alignment and user friendliness. This will open new fast modalities as XRF tomography and XANES imaging for users.

[1] A. Somogyi et al, J. Synch. Rad. 22 (2015) p. 1118.

[2] A. Singer \& A. Vartanyants, J. Synch. Rad. 21 (2014) p. 5.

[3] C. Schroer \& , J. Synch. Rad. 21 (2014) p. 996.

[4] M. D. de Jonge et al, J. Synch. Rad. 21 (2014) p. 1031. 